\title{
Identification of the Active Constituents and Significant Pathways of Shen-qi-Yi-zhu Decoction on Antigastric Cancer: A Network Pharmacology Research and Experimental Validation
}

\author{
ShuhongZeng $\mathbb{D}^{1,2}$ Zhibao Yu ${ }^{10},{ }^{1,2}$ Xintian Xu, ${ }^{1,2}$ Yuanjie Liu, ${ }^{1,2}$ Jiepin Li, ${ }^{1,2,3}$ Danya Zhao, ${ }^{4}$ \\ Changjuan Song, ${ }^{1,2}$ Haixia Lu, ${ }^{1,2}$ Yudong Zhao, ${ }^{1}$ Weimin Lu $\mathbb{D}^{1},{ }^{1}$ and Xi Zou $\mathbb{D}^{1}$ \\ ${ }^{1}$ Affiliated Hospital of Nanjing University of Chinese Medicine, Jiangsu Province Hospital of Chinese Medicine, Nanjing, \\ Jiangsu 210029, China \\ ${ }^{2}$ No. 1 Clinical Medical College, Nanjing University of Chinese Medicine, Nanjing, Jiangsu 210023, China \\ ${ }^{3}$ Department of Oncology, Zhangjiagang TCM Hospital Affiliated to Nanjing University of Chinese Medicine, Zhangjiagang, \\ Jiangsu 215600, China \\ ${ }^{4}$ The First School of Clinical Medicine, Zhejiang Chinese Medicine University, Hangzhou, Zhejiang 310053, China \\ Correspondence should be addressed to Weimin Lu; wmlu@163.com and Xi Zou; zxvery@126.com
}

Received 11 October 2020; Accepted 13 October 2021; Published 22 November 2021

Academic Editor: Hua Li

Copyright ( $\odot 2021$ Shuhong Zeng et al. This is an open access article distributed under the Creative Commons Attribution License, which permits unrestricted use, distribution, and reproduction in any medium, provided the original work is properly cited.

\begin{abstract}
Shen-qi-Yi-zhu decoction (SQYZD) is an empirical prescription with antigastric cancer (GC) property created by Xu Jing-fan, a National Chinese Medical Master. However, its underlying mechanisms are still unclear. Based on network pharmacology and experimental verification, this study puts forward a systematic method to clarify the anti-GC mechanism of SQYZD. The active ingredients of SQYZD and their potential targets were acquired from the TCMSP database. The target genes related to GC gathered from GeneCards, DisGeNET, OMIM, TTD, and DrugBank databases were imported to establish protein-protein interaction (PPI) networks in GeneMANIA. Cytoscape was used to establish the drug-ingredients-targets-disease network. The hub target genes collected from the SQYZD and GC were parsed via GO and KEGG analysis. Our findings from network pharmacology were successfully validated using an in vitro HGC27 cell model experiment. In a word, this study proves that the combination of network pharmacology and in vitro experiments is effective in clarifying the potential molecular mechanism of traditional Chinese medicine (TCM).
\end{abstract}

\section{Introduction}

Gastric cancer (GC) is one of the most common causes of cancer-related mortality worldwide [1]. Currently, the main problems of gastric cancer treatment are drug resistance and complications after surgery, chemotherapy, and radiotherapy [2]. In recent years, great progress has been made in the treatment of tumors by traditional Chinese medicine (TCM) and TCM combined with chemotherapy [3]. Shen-qi-Yi-zhu decoction (SQYZD) was created by National Chinese Medical Master Xu Jing-fan. He is experienced in the diagnosis and treatment of digestive system diseases and has been engaged in TCM clinical practice for more than 60 years.
Several studies have shown that SQYZD is effective in relieving symptoms and improving GC patients' quality of life. A study by Xu found that SQYZD can reduce chemotherapy-induced side effects such as myelosuppression (thrombocytopenia, hemoglobinemia, and leucopenia), gastrointestinal reactions (nausea, vomiting, and diarrhea), and peripheral neurotoxicity [4]. SQYZD can inhibit the transformation, invasion, and metastasis of gastric cancer cells by inhibiting the $\mathrm{Wnt} / \beta$-catenin signaling pathway [5]. Another study by Sun found that SQYZD combined with chemotherapy can improve patients' symptoms such as stomachache, epigastric puffiness, poor appetite, pantothenic acid, loose stool, and fatigue., improve body function, reduce the adverse reactions caused by 
chemotherapy (nausea, vomiting, and diarrhea), and improve the quality of life of patients after gastric cancer [6]. Therefore, it is necessary to clarify the biological basis and molecular mechanism of the SQYZD. In recent years, with the development of network pharmacology, combined with its characteristics of "Drug-component-target-disease," it can provide a new idea for the research of disease treatment and drug selection [7].

In this study, a network pharmacology approach was used to explore the potential mechanism of SQYZD in treating GC. Based on some results from network pharmacology, relevant experiments were designed for validation.

\section{Materials and Methods}

\subsection{Network Pharmacology}

2.1.1. Screening of SQYZD for Active Ingredients and Corresponding Targets. SQYZD was composed by the following herbs: Hedysarum Multijugum Maxim (Huang-qi), Codonopsis Radix (Dang-shen), Coicis Semen (Yi-yi-ren), Atractylodes Macrocephala Koidz (Bai-zhu), Curcumae Rhizoma (E-zhu), Poria Cocos (Schw.) Wolf (Fu-ling), Hedyotis Diffusae Herba (Bai-hua-she-she-cao), Agrimonia Eupatoria (Xian-he-cao), and Licorice (Gan-cao).

The chemical constituents of SQYZD were accessed through the Traditional Chinese Medicine Systems Pharmacology database (TCMSP; https://www.tcmsp-e.com/) [8]. The bioactive compounds were retrieved under the retrieval filters of oral bioavailability $(\mathrm{OB}) \geq 30 \%$ and drug-likeness $(\mathrm{DL}) \geq 0.18[9,10]$. The TCMSP database was also the main source of component-target data. We converted the target names of the bioactive components of SQYZD into gene names with the species limited to "Homo sapiens" with the UniProt Knowledgebase (https://www.uniprot.org/) [11].

2.1.2. Identification of Anti-GC Targets. To find the relevant targets comprehensively, information on GC-associated target genes was combined from the following 5 databases: Genecards (https://www.genecards.org), DisGeNET (https://www.disgenet.org/home/), Online Mendelian Inheritance in Man (OMIM; https://omim.org), Therapeutic Target Database (TTD; http://db.idrblab.net/ttd/), and DrugBank (https://www.drugbank.ca) [12-16]. By combining the genes obtained from the 5 databases after removing the duplicates, protein targets associated with GC were identified. The obtained targets were standardized through the UniProt Knowledgebase as mentioned above.

\subsubsection{Protein-Protein Interaction (PPI) Network Analysis and} Drug-Ingredients-Targets-Disease Network Construction. The overlapping target proteins of GC and SQYZD were used to construct a PPI network with multiple protein patterns on the GeneMANIA platform (http://genemania. org/) [17]. We set the organism type to "Homo sapiens" and left the default settings in place for the other parameters on the Search Tool for the Retrieval of Interacting Genes/
Proteins (STRING) platform (https://string-db.org/, version 11.5) [18]. Then, we imported the information on protein interactions into Cytoscape 3.7.2 (Cytoscape Software, Inc., USA) to obtain the drug-ingredients-targets-disease network and perform network analysis [19]. In the network, nodes represent the target proteins, and edges represent the interactions between proteins.

2.1.4. GO and KEGG Pathway Analysis. The Gene Ontology (GO) and Kyoto Encyclopedia of Genes and Genomes (KEGG) pathway enrichment analysis are important methods used to describe the characteristics of candidate targets $[20,21]$. We selected the standard $p$ value cut off of 0.05 and the $q$ value of 0.05 and performed the enrichment analysis with RStudio 3.6.1 (Bioconductor, clusterProfiler) [22].

\subsection{Experimental Validation}

2.2.1. Preparation of SQYZD. All herbs were purchased from Zhongshan Chinese Traditional Medicine Co. (Nanjing, China) and were identified by a Chinese pharmacist. All of the herbs were soaked for $30 \mathrm{~min}$ with $1000 \mathrm{ml}$ of doubledistilled water, and then, boiled on a medium fire for $30 \mathrm{~min}$, refluxed and extracted. The boiling process was repeated with another $1500 \mathrm{ml}$ of double-distilled water for $30 \mathrm{~min}$. The two extracted solutions were then mixed and further vaporized to $50 \mathrm{ml}$ by boiling. The concentration of raw herbs was $2 \mathrm{~g} / \mathrm{ml}$. Sodium bicarbonate was used to correct the $\mathrm{pH}$ value of the SQYZD concentration. The extract was stored at $-20^{\circ} \mathrm{C}$ after sterilization and filtered through a $0.22 \mu \mathrm{m}$ filter.

2.2.2. Mass Spectrometry Analysis. To analyze and identify the chemical constituents in the extracts of SQYZD, we conducted a mass spectrometry analysis. $1 \mathrm{ml}$ of $80 \%$ methanol was added to $200 \mu \mathrm{l}$ SQYZD and vortexed for $5 \mathrm{~min}$. Then, it was centrifuged at $4^{\circ} \mathrm{C}$ for $10 \mathrm{~min}$ at $12000 \mathrm{rpm}$. The supernatant was taken for ESI-Q-ExactiveOrbitrap MS in positive/negative ion mode (Thermo Fisher, USA). The data collected by high-resolution liquid quality were sorted preliminarily by CD 2.1 (Thermo Fisher, USA) and then retrieved and compared in MzCloud database.

2.2.3. Cell Culture. HGC27 (undifferentiated GC cells) was purchased from the Chinese National Collection of $\mathrm{Au}-$ thenticated Cell Cultures (Shanghai, China). HGC27 was cultured in RPMI-1640 medium (Gibco, USA) with 10\% fetal bovine serum (FBS, Every Green, China), $100 \mathrm{U} / \mathrm{ml}$ penicillin, and $100 \mathrm{mg} / \mathrm{ml}$ streptomycin (Gibco, USA) and was incubated at $37^{\circ} \mathrm{C}$ in $5 \% \mathrm{CO}_{2}$.

2.2.4. Cell Counting Kit-8 (CCK-8) Assay. CCK-8 was used to assess the cytotoxicity of SQYZD. After HGC27 cells were seeded in a 96-well culture plate (Corning, USA) at a density of $2 \times 10^{3}$ cells/well for $24 \mathrm{~h}$, various concentrations of SQYZD $(0,1.25,2.5,5.0,7.5,10$, and $12 \mathrm{mg} / \mathrm{ml})$ were used to 
incubate with the cells. Subsequently, $10 \mu \mathrm{l}$ CCK-8 solution (Dojindo, Japan) was applied to each well, followed by incubation for $3 \mathrm{~h}$ at $37^{\circ} \mathrm{C}$ in $5 \% \mathrm{CO}_{2}$. Finally, the absorbance was measured at $450 \mathrm{~nm}$ on a microplate reader. The relative cell viability rate was calculated as follows: (treated/control) $\times 100 \%$. The $\mathrm{IC}_{50}$ values were obtained using the logit method.

2.2.5. Colony Formation Assay. We assessed the clonogenic ability of cells using a clone formation assay. $2 \times 10^{3}$ cells/ well were seeded in a 6-well plate (Corning, USA). After attachment, the cells were treated with/without different concentrations of SQYZD $(0,2,4$, and $8 \mathrm{mg} / \mathrm{ml})$ and were cultured with a new medium for 14 days after the SQYZDcontaining medium was discarded. After fixation with $4 \%$ paraformaldehyde for $15 \mathrm{~min}$, the cells were stained with crystal violet solution for $10 \mathrm{~min}$ at room temperature. Finally, the plate was washed moderately with water, and the colony with at least 100 cells was counted. Digital photography of colonies was conducted.

2.2.6. TUNEL Staining. The TUNEL assay was used to assess the cell apoptosis. According to the instructions of the manufacturer, DNA fragments were determined using a One Step TUNEL Apoptosis Assay Kit (Beyotime, China). The nucleus was also stained with DAPI (Beyotime, China). TUNEL-negative (blue) and TUNEL-positive (red) cells were observed using a fluorescence microscope (NIKON DS-Qi2, $\times 200$ magnification).

2.2.7. Wound Healing Assay. Cells were inoculated into 6well plates (Corning, USA), such that the cell density reached about $60 \%$. After cell adhesion, the medium was discarded, and three lines were drawn on the cell monolayer using a sterile $10-\mu \mathrm{l}$ pipette tip by following the lines present at the bottom of the plate, with three secondary wells for each sample. Scratches were observed after incubations for 12 hours, 24 hours, and 48 hours in $5 \% \mathrm{CO}_{2}$ at $37^{\circ} \mathrm{C}$ and were photographed under a microscope (Olympus CKX-41, $\times 200$ magnification).

2.2.8. Transwell Migration Assay. Cell migration analysis was performed using migration chambers. Equal numbers $\left(5 \times 10^{3}\right.$ cell/well $)$ of HGC27 cells in serum-free medium were seeded in the upper chamber $(8 \mu \mathrm{m}, 24$-well, Corning, USA) and treated with/without different concentrations of $\operatorname{SQYZD}(0,2,4$, and $8 \mathrm{mg} / \mathrm{ml})$. A normal medium containing $10 \%$ FBS was added to the lower chamber of a 24-well plate as an attractant. The cells were cultured for 24 hours, then cells remaining on the upper surface of the membrane were removed, while cells that migrated to the lower surface of the membrane were fixed in $4 \%$ paraformaldehyde for $15 \mathrm{~min}$ and stained with crystal violet. Five random fields were photographed under a microscope (Olympus CKX-41, ×200 magnification), and the cell number was counted using Image J software (Image J Software, Inc., USA).
2.2.9. Western Blot Assessment. Whole cell lysates were extracted from HGC27 cells treated with/without different concentrations of SQYZD $(0,2,4$, and $8 \mathrm{mg} / \mathrm{ml})$ and IGF-1 (insulin-like growth factor-1, PEPRO Tech, $5 \mu \mathrm{M}$ ), a growth factor as an agonist to PI3K/AKT/mTOR signaling pathway, using a standard protocol with RIPA lysis buffer (Beyotime, China). The cell lysate samples $(10 \mu \mathrm{g})$ were electrophoresed in $10 \%$ sodium dodecyl-sulfate polyacrylamide gel (SDSPAGE) and then transferred to PVDF membranes $(0.2 \mu \mathrm{m}$, Merck Millipore, USA). The membranes were then blockedin blocking buffer (NCM Biotech, China) for $10 \mathrm{~min}$ at room temperature and incubated at $4^{\circ} \mathrm{C}$ overnight with primary antibodies. After washing three times, the membranes were incubated with HRP-conjugated secondary antibodies. Target $/ \beta$-actin bands were identified with a gel image processing system (ChemiDoc XRS+). Subsequently, relative protein levels were calculated.

2.2.10. Statistical Analysis. Data were analyzed by GraphPad Prism 8.0 (GraphPad Software, Inc., USA) and reported as means \pm standard deviation. We used one-way ANOVA followed by Dunnett's t-test to compare between two groups and multiple groups, respectively. Values of $p<0.05$ were considered statistically significant. All experiments were conducted at least three times.

\section{Results}

\subsection{Network Pharmacology}

3.1.1. Active Ingredients of SQYZD. A total of 787 active ingredients of the nine herb medicines in SQYZD were retrieved from TCMSP, including 87 ingredients in Hedysarum Multijugum Maxim (Huang-qi), 134 ingredients in Codonopsis Radix (Dang-shen), 38 ingredients in Coicis Semen (Yi-yi-ren), 55 ingredients in Atractylodes Macrocephala Koidz (Bai-zhu), 81 ingredients in Curcumae Rhizoma (E-zhu), 34 ingredients in Poria Cocos (Schw.) Wolf. (Fu-ling), 37 ingredients in Hedyotis Diffusae Herba (Baihua-she-she-cao), 41 ingredients in Agrimonia Eupatoria (Xian-he-cao), and 280 ingredients in Licorice (Gan-cao). 179 ingredients passed the filters of $\mathrm{OB} \geq 30 \%$ and $\mathrm{DL} \geq 0.18$. Finally, 161 active ingredients passed the repetition. The compounds are shown in Supplement 1.

3.1.2. Identification of Candidate Targets of SQYZD for GC Treatment. After the removal of duplicate targets, a total of 2126 GC-related targets were identified from Genecards, DisGeNET, OMIM, TTD, and DrugBank. The targets were correlated, and a total of 87 targets were indicated as the potential targets with Venny (https://bioinfogp.cnb.csic.es/ tools/venny/) (Figure 1(a)).

3.1.3. PPI Network Analysis and Drug-Ingredients-TargetsDisease Network Construction. The 87 common targets were imported to the GeneMANIA database to establish the forecast target PPI network (Figure 1(b)). The confidence level was set to be greater than 0.900 , and isolated target 

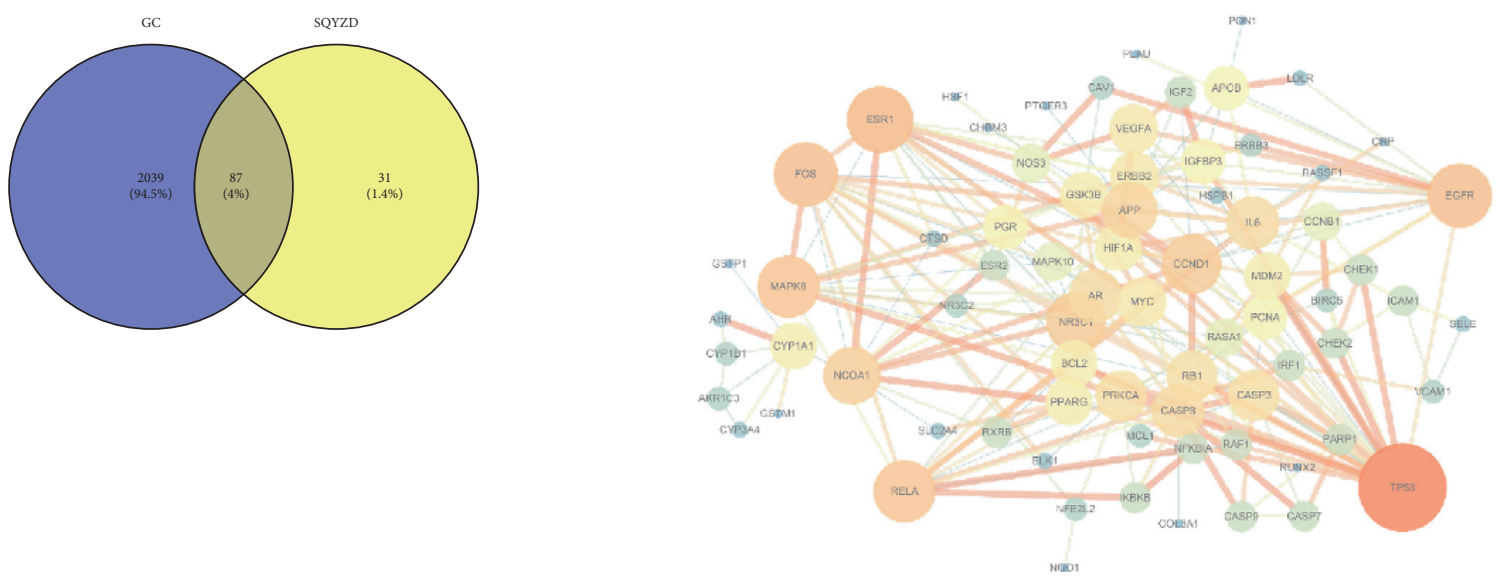

(a)

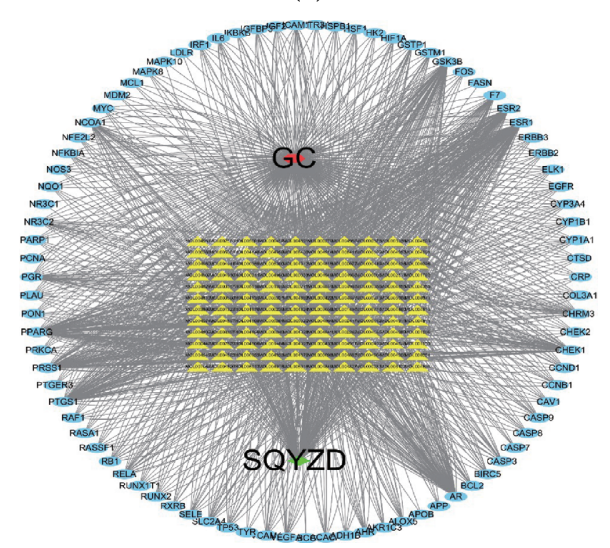

(c)

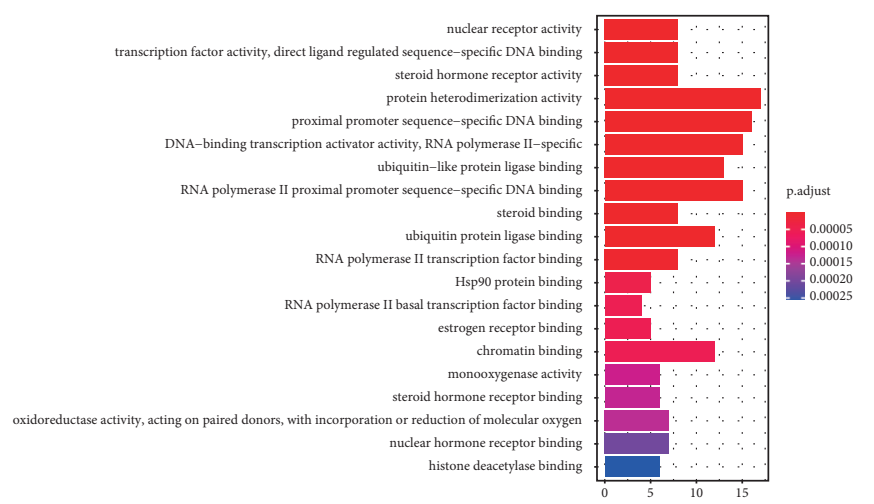

(d)

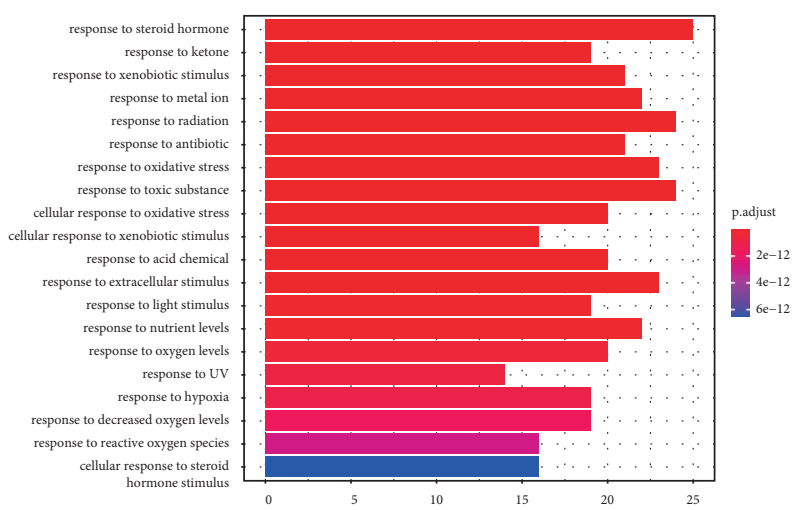

(e)

Figure 1: Continued. 


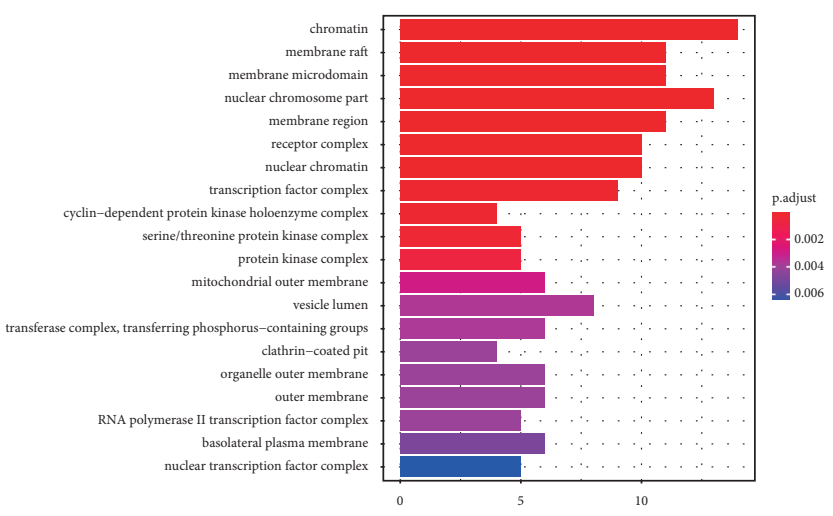

(g)

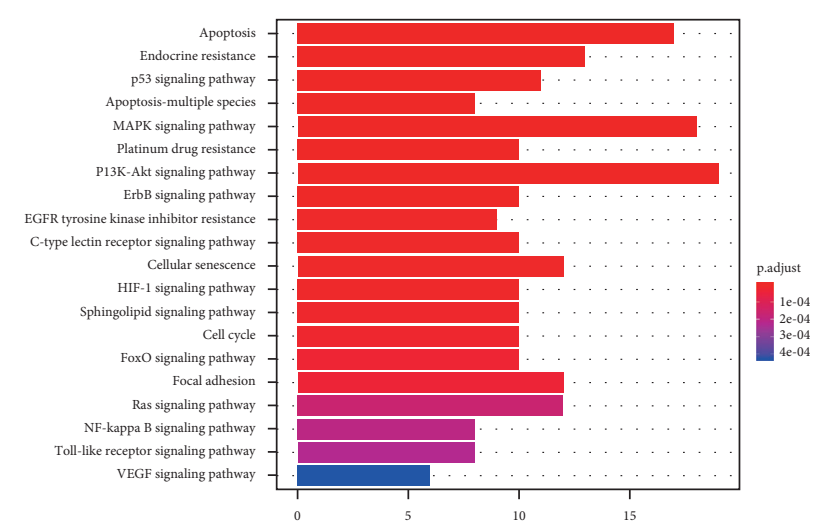

(h)

FIgURE 1: (a) The overlap targets with Venny; (b) the PPI network of targets for SQYZD in treating GC (from dark to light, from small to large, the degrees of freedom increase, and the thicker edges suggest stronger interactions); (c) the top 30 of the core genes; and (d) the SQYZD-GC-related drug targets. The red rectangle, green rectangle, yellow rectangle, and blue rectangle represent disease, herbs, the common targets between SQYZD and GC, and the active ingredient of SQYZD. (e-h) GO and KEGG enrichment analysis and therapeutic targets of SQYZD in treating GC. (e) MF; (f) BP; (g) CC; and (h) KEGG.

proteins were eliminated to obtain protein interaction information. We imported the protein interaction information into Cytoscape 3.7.2 to obtain drug-ingredients-targets-disease network analysis. The network had 75 nodes and interacted with 212 edges. We obtained the core genes of the top 30 (Figure $1(\mathrm{c})$ ). The targets of 87 therapeutic targets for the treatment of GC include TP53, ERS1, EGFR, FOS, MAPK8, RELA, CCND1, CASP8, IL-6, CASP3, HIF1A, MDM2, and BCL2. These results demonstrated that these targets were closely related to others in the network and, consequently, might play key roles in GC (Figure $1(\mathrm{~d})$ ).

3.1.4. GO Enrichment and KEGG Pathway Analysis. To further illuminate the biological effects involved in the treatment of GC with SQYZD, we performed GO analysis of the 87 GC-related target genes. GO annotation and enrichment of the genes encoding SQYZD were conducted from three aspects: molecular function (MF), biological process (BP), and cellular composition (CC). The KEGG pathway enrichment analysis was also carried out. The most enriched terms with GO and KEGG pathway analysis are shown in Figures 1(e)-1(h).

In detail, the top 10 terms of the GO BP category were mainly enriched in response to steroid hormones, response to radiation, response to toxic substances, response to extracellular stimulus, response to oxidative stress, response to metal ions, response to nutrient levels, response to xenobiotic stimulus, response to antibiotics, and cellular response to oxidative stress. The top 10 terms in the GO CC category were mainly enriched in chromatin, nuclear chromosome part, membrane raft, membrane microdomain, membrane region, receptor complex, nuclear chromatin, transcription factor complex, serine/threonine protein kinase complex, and protein kinase complex. The top 10 terms of the GO MF category were mainly enriched in protein heterodimerization activity, proximal promoter sequence-specific DNA binding, DNA-binding transcription activator activity, RNA polymerase II-specific, RNA polymerase II proximal promoter, sequence-specific DNA binding, ubiquitin-like protein ligase binding, ubiquitin-protein ligase binding, chromatin binding, nuclear receptor activity, transcription factor activity, direct ligand regulated sequence-specific DNA binding, and steroid hormone receptor activity.

According to the $p$ value, the PI3K-AKT signaling pathway, the MAPK signaling pathway, apoptosis, endocrine resistance, cellular senescence, the P53 signaling pathway, platinum drug resistance, the $\operatorname{ErbB}(\mathrm{Her})$ signaling pathway, the HIF-1 signaling pathway, and the FoxO signaling pathway were within the top 10 pathways.

\subsection{In Vitro Experiments}

3.2.1. Mass Spectrometry Result of SQYZD. The results of ESI-Q-Exactive-Orbitrap MS in positive/negative ion mode are shown in Figure 2(a). A total of 258 compounds were matched in mzCloud for the SQYZD sample. 63 compounds with best match score were over 70 in mzCloud (Supplement 2). The 32 compounds were both in the results of the network pharmacology and the ESI-Q-Exactive-Orbitrap MS.

3.2.2. SQYZD Inhibits the Growth of HGC27 and Induces Apoptosis. SQYZD significantly inhibited the cell proliferation of HGC27. After intervention with SQYZD for $48 \mathrm{~h}$, the $\mathrm{IC}_{50}$ was $7.851 \mathrm{mg} / \mathrm{ml}$ (Figure 2(b)). Besides, the number of colonies formed by HGC27 decreased with an increase of SQYZD concentration (Figure 2(c)).

With the increase of SQYZD concentration, the TUNELpositive cells increased gradually (Figure 2(d)). Western blotting of classical apoptotic molecule also revealed that with the increase of SQYZD concentration, the degree of apoptosis of HGC27 cells increased $(p<0.05)$ (Figures 2(e) and $2(\mathrm{f})$ ). The degree of apoptosis in all treatment groups was higher than that in the control group, and it was concentration-dependent. The results of in vitro experiments 


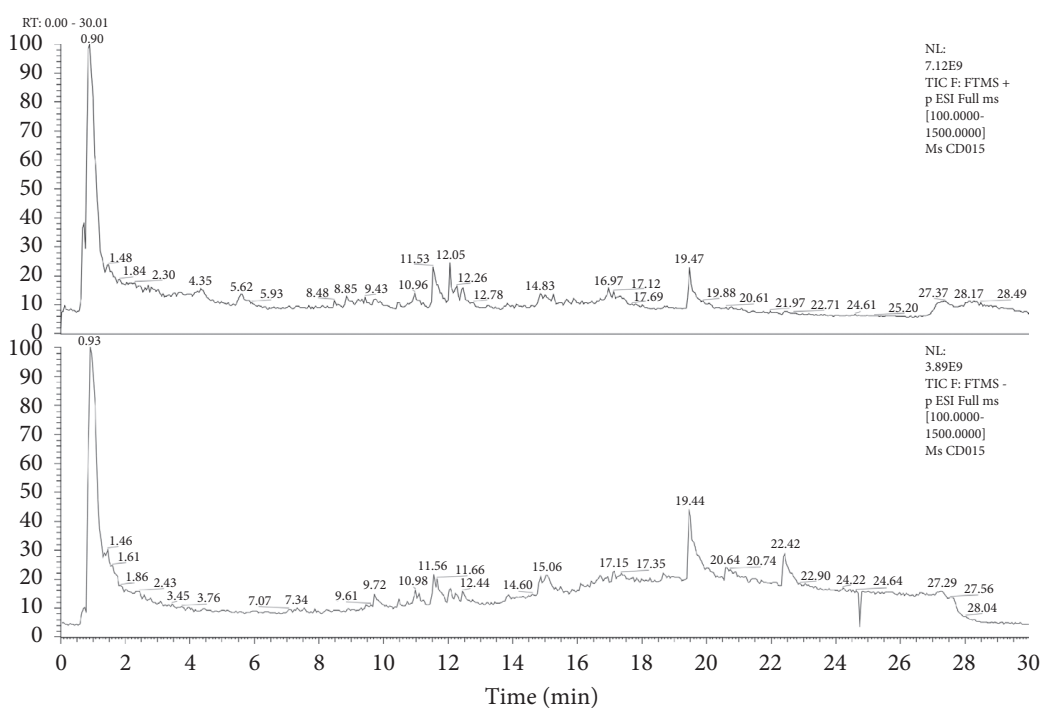

(a)

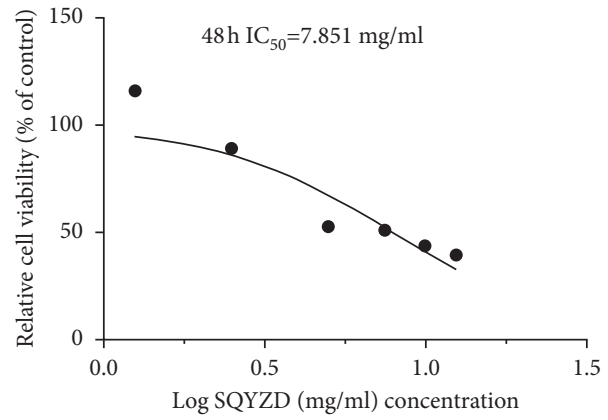

(b)

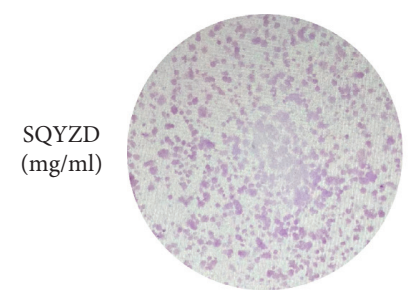

0

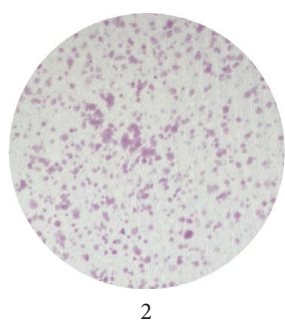

(c)

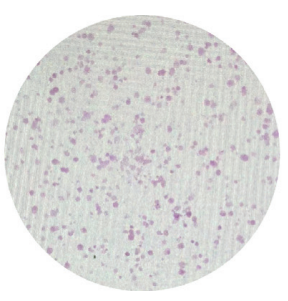

4

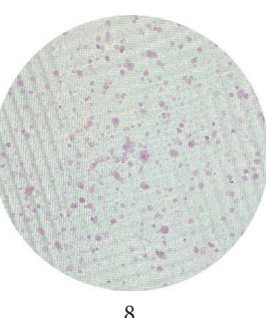

8

Figure 2: Continued. 

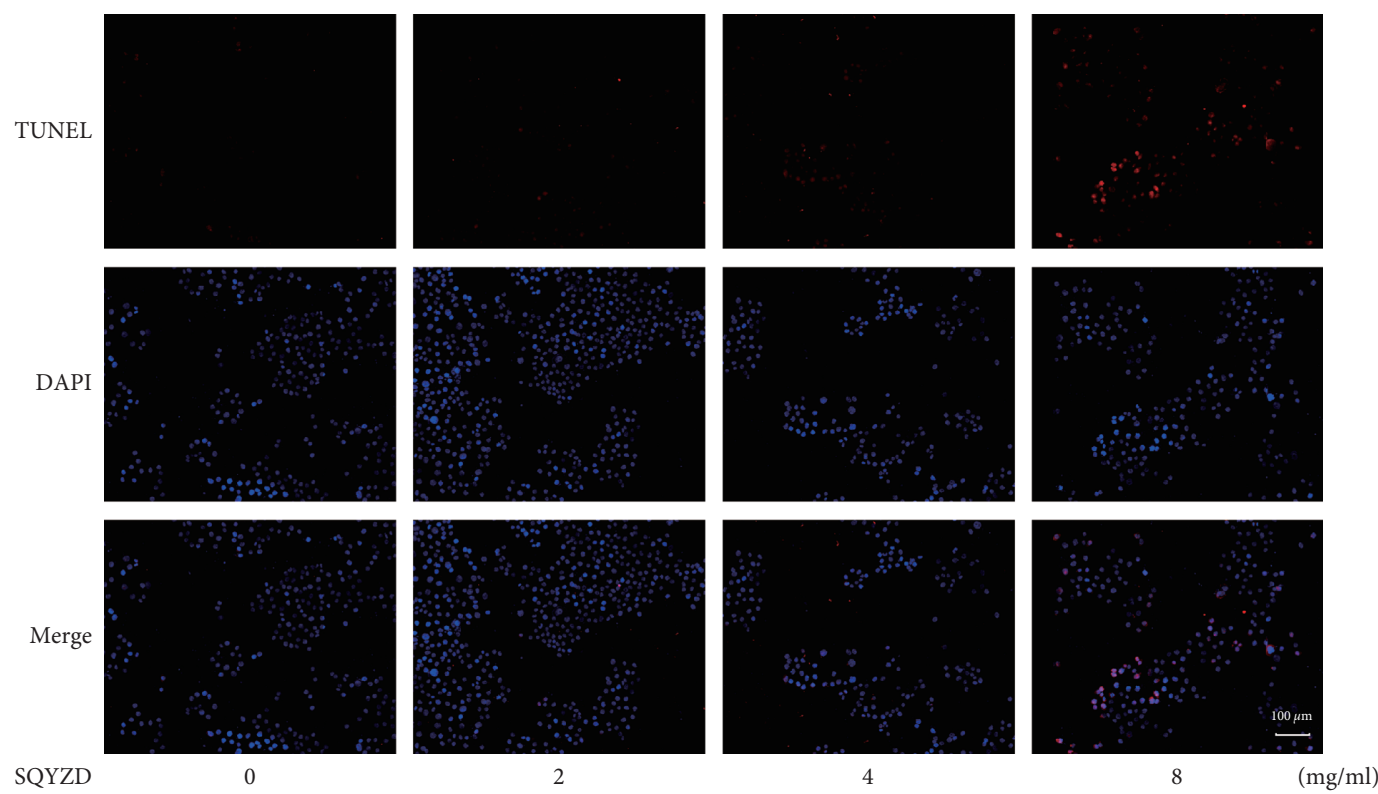

(d)

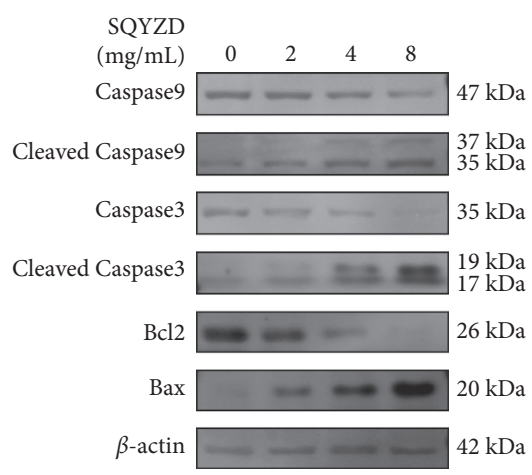

(e)
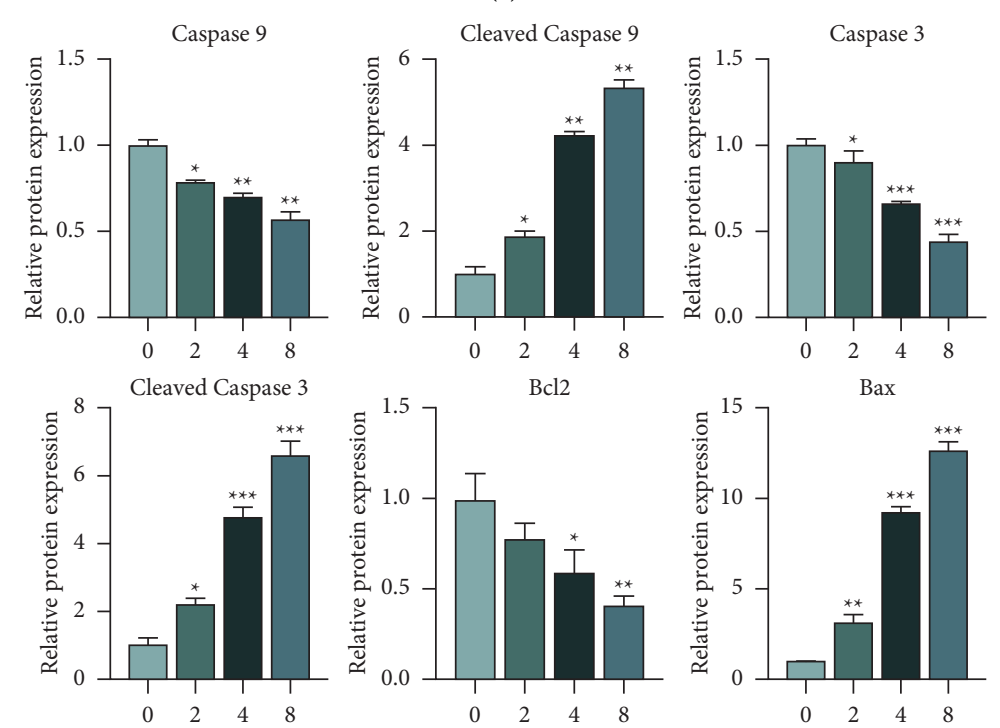

(f)

FIgURE 2: (a) The results of ESI-Q-Exactive-Orbitrap MS in positive/negative ion mode; (b) relative cell viability of HGC27 cells treated with/without SQYZD $(0,2,4$, and $8 \mathrm{mg} / \mathrm{ml})$; (c) colony formation capacity of the HGC27 cells treated with/without SQYZD (0, 2, 4, and $8 \mathrm{mg} / \mathrm{ml}$ ); (d) apoptosis assessed using a TUNEL assay (TUNEL-positive cells indicated in red) (magnification, $\times 200$ ); (e) and (f) Expression of apoptosis-related proteins examined using western blots after treatment with/without SQYZD (0, 2, 4, and $8 \mathrm{mg} / \mathrm{ml})$. 


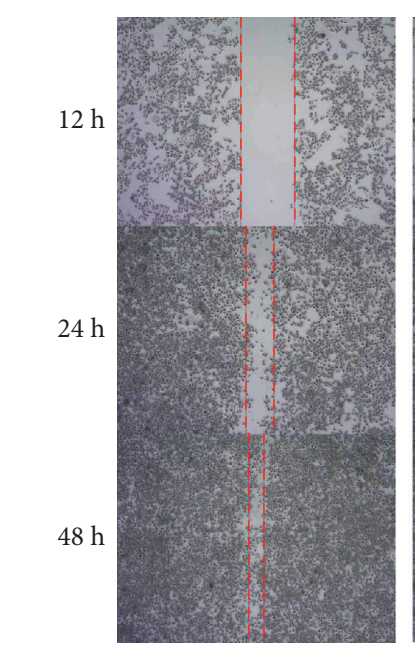

SQYZD

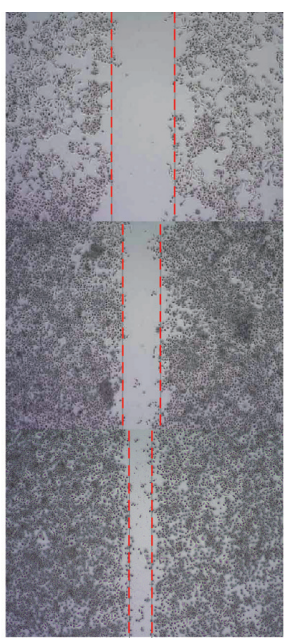

2

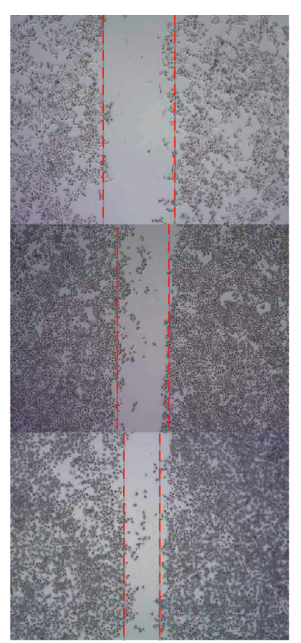

4

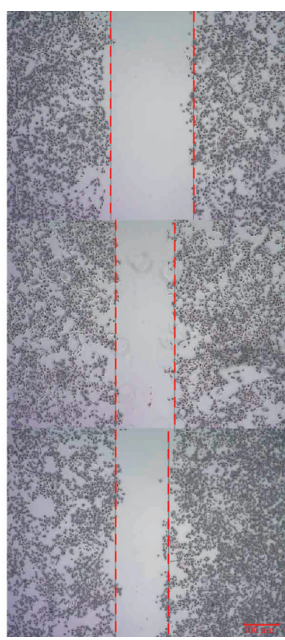

$8 \quad(\mathrm{mg} / \mathrm{ml})$

(a)

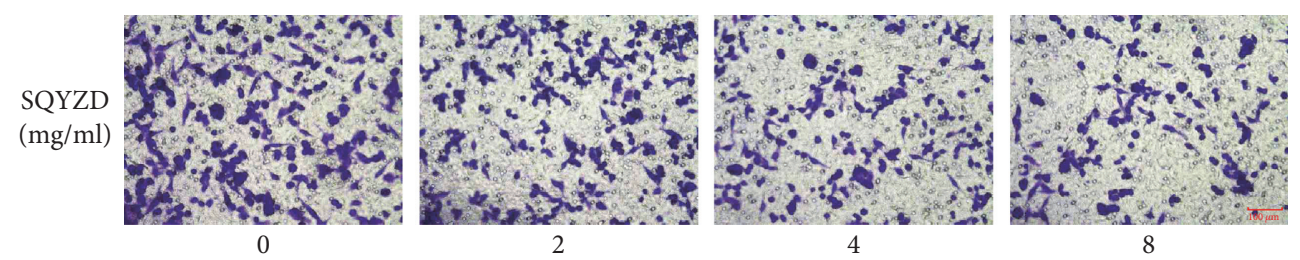

(b)

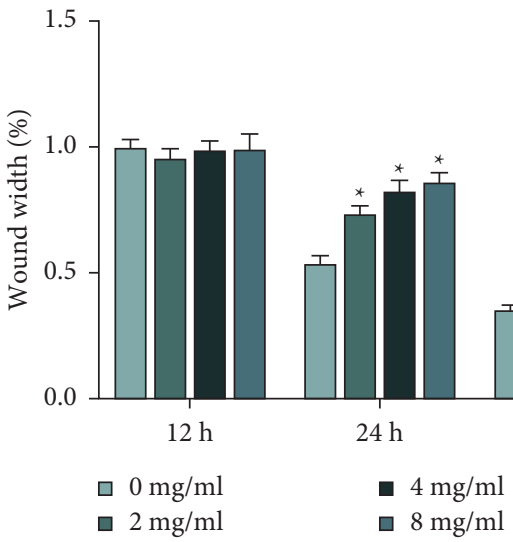

(c)

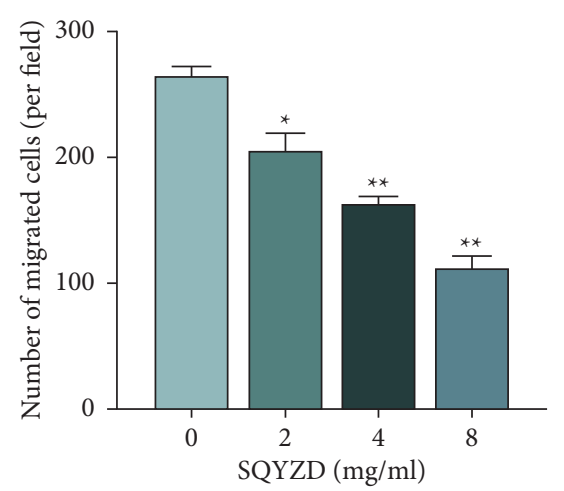

(d)

Figure 3: Continued. 


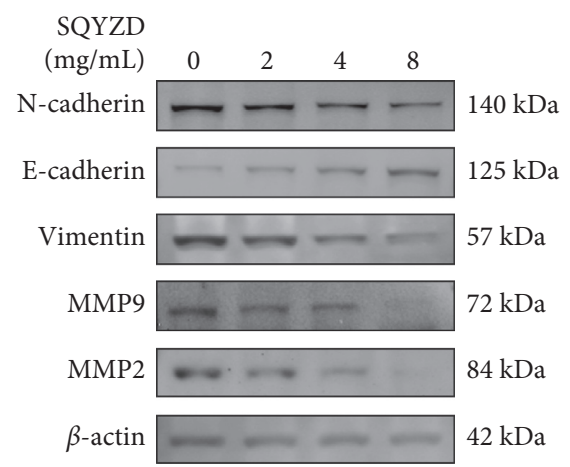

(e)
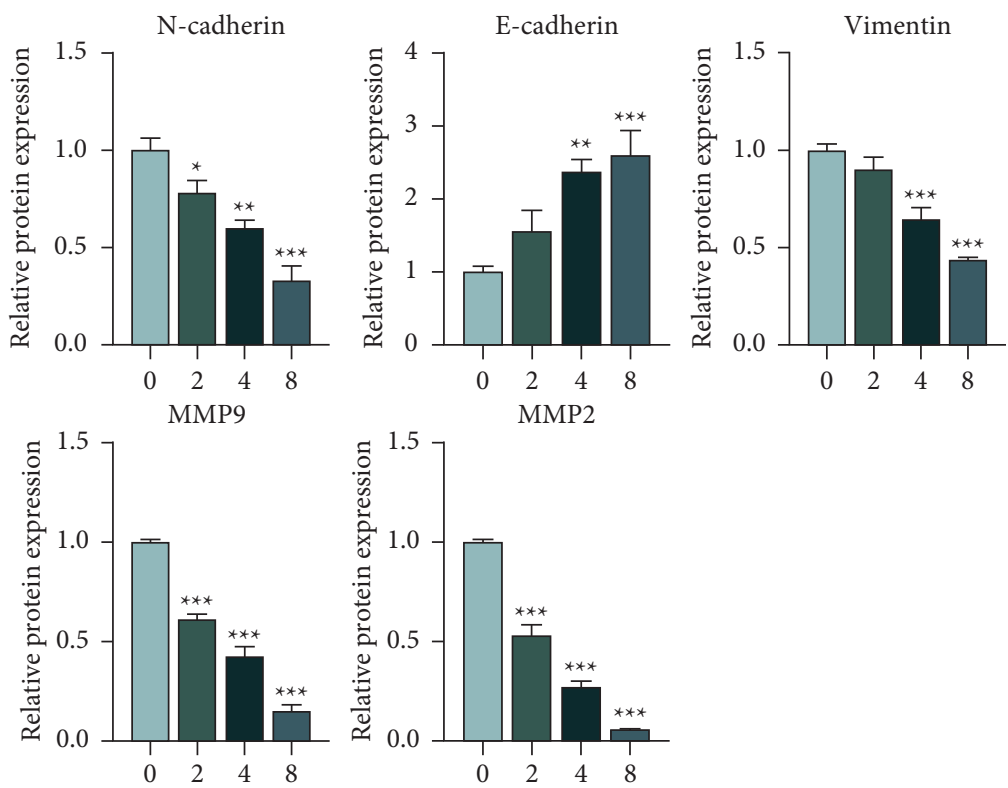

(f)

FIgURE 3: (a) Migratory ability of different groups of HGC27 cells treated with/without SQYZD (0, 2, 4, and $8 \mathrm{mg} / \mathrm{ml}$ ) examined using wound healing assays. (c) The percentages of wound width at $12 \mathrm{~h}, 24 \mathrm{~h}$, and $48 \mathrm{~h}$. (b) The migration ability of GC cells after treatment with/ without SQYZD $(0,2,4$, and $8 \mathrm{mg} / \mathrm{ml})$. (d) The relative migratory cell number. (e, f) Expression of EMT-related proteins examined using western blots after treatment with/without SQYZD $(0,2,4$, and $8 \mathrm{mg} / \mathrm{ml})$.

were consistent with the conclusions of network pharmacology.

\subsubsection{SQYZD Suppresses the Epithelial-Mesenchymal} Transition (EMT) of HGC27. An increase in SQYZD concentration significantly inhibited cell wound healing (Figures 3(a) and 3(c)) and migration (Figures 3(b) and $3(d))$. Moreover, an increase of SQYZD concentration induced E-cadherin while inhibited N-cadherin, vimentin, and MMP2 and MMP9 levels $(p<0.05)$ (Figures 3(e) and 3(f)). These data indicated that SQYZD inhibited EMT in GC.

3.2.4. SQYZD Inhibits $P I 3 K / A K T / m T O R$ Signaling in $G C$. To further explore the potential mechanisms of anti-GC, according to the results of KEGG pathway analysis in network pharmacology, we first analyzed the effect of SQYZD on the expression of key molecules in the PI3K/ $\mathrm{AKT} / \mathrm{mTOR}$ axis. Increase of SQYZD concentration inhibited mTOR, PI3K, p-PI3K, AKT, and p-AKT levels $(p<0.05)$ (Figures $4(\mathrm{a})$ and $4(\mathrm{~b}))$.

In order to further confirm our idea, we conducted verification experiments with IGF-1 (a growth factor as the agonist of PI3K/AKT/mTOR). We observed that mTOR, $\mathrm{PI} 3 \mathrm{~K}, \mathrm{p}-\mathrm{PI} 3 \mathrm{~K}, \mathrm{AKT}$, and p-AKT levels were significantly in the IGF-1 group $(p<0.05)$. After treating with SQYZD, mTOR, PI3K, p-PI3K, AKT, and p-AKT levels were downregulated in HGC27 cells (Figures 4(c) and 4(d)).

These data indicated that SQYZD suppressed proliferation, antiapoptosis, and EMT might be through inhibiting the PI3K/AKT/mTOR signaling pathway in vitro.

\section{Discussion}

In recent years, TCM has played a unique role in the antitumor area. TCM can reduce the toxic and side effects of chemotherapy and improve the quality of GC patients' lives $[23,24]$. However, due to the complex chemical composition 

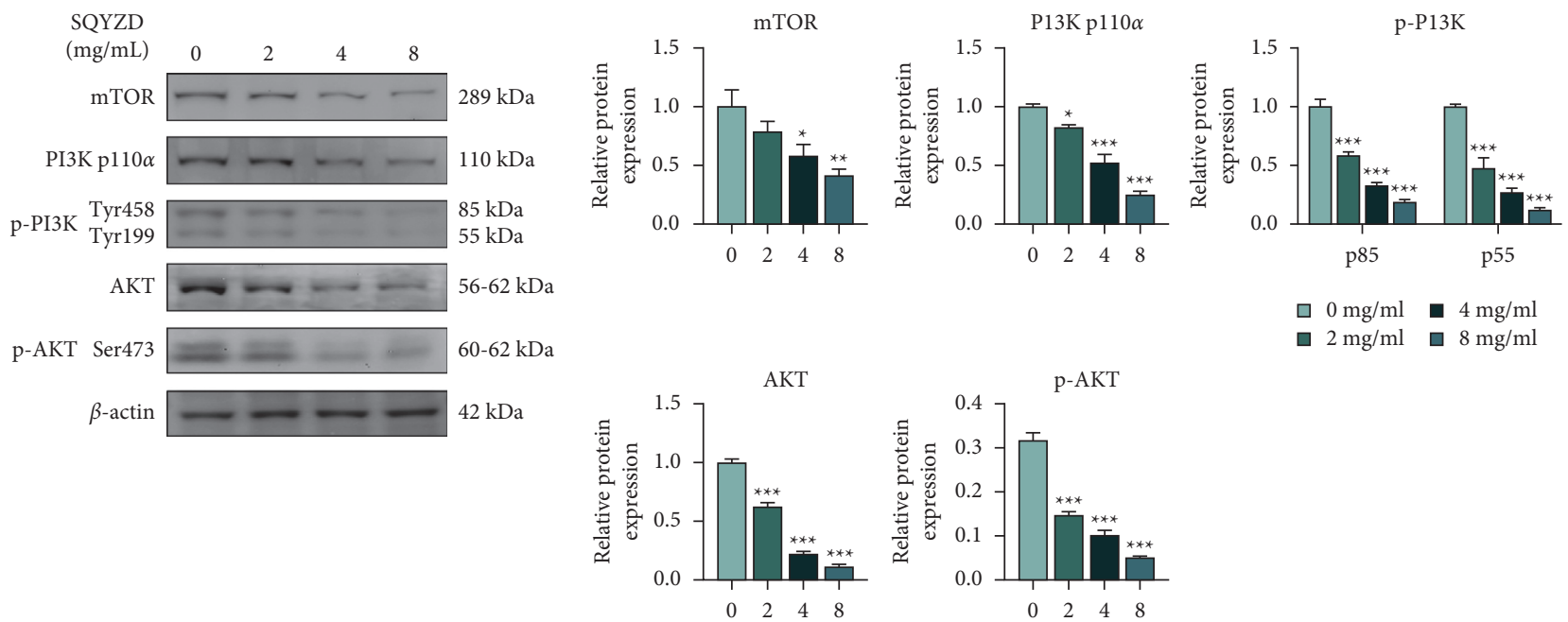

(a)
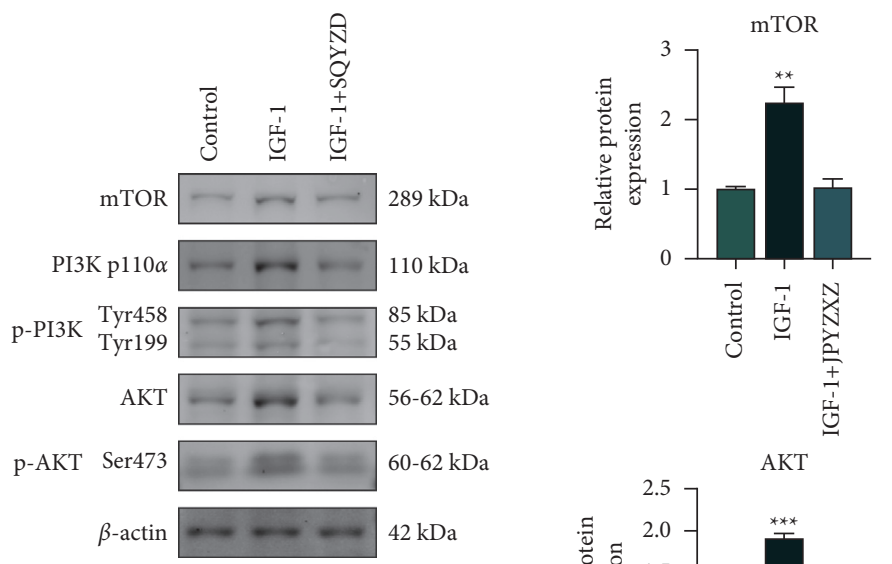

(b)
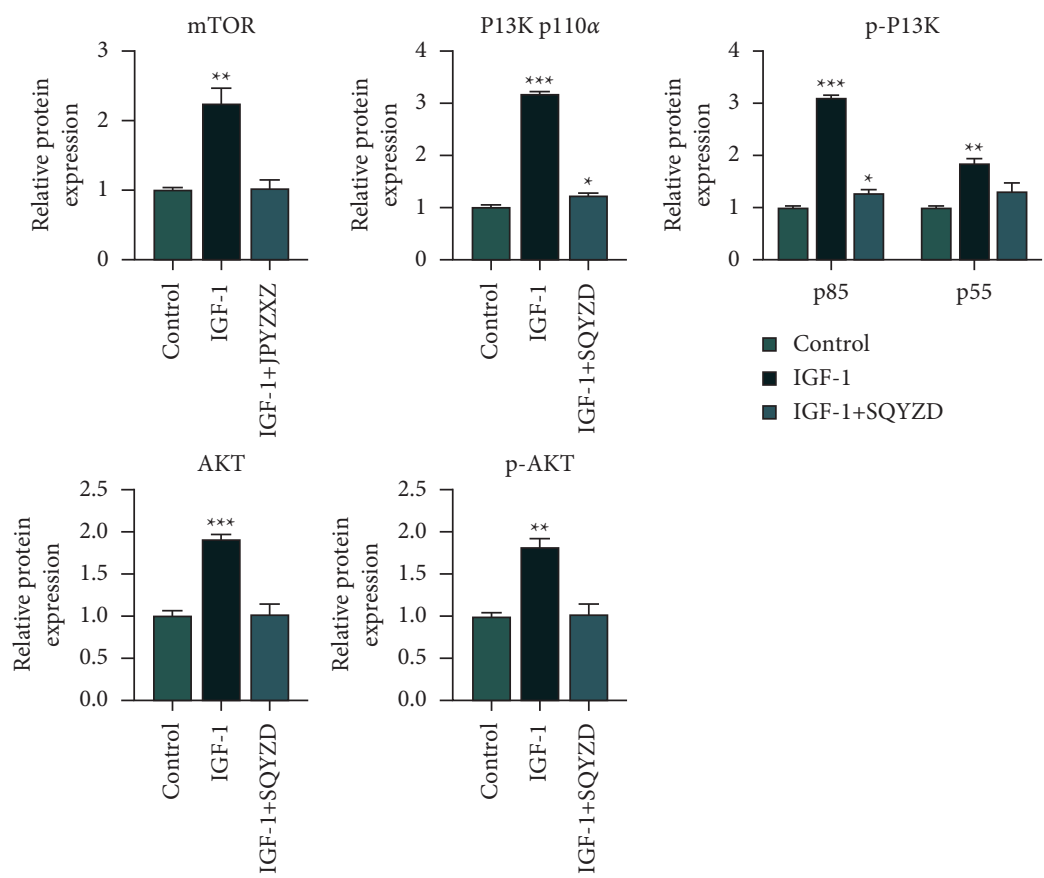

(c)

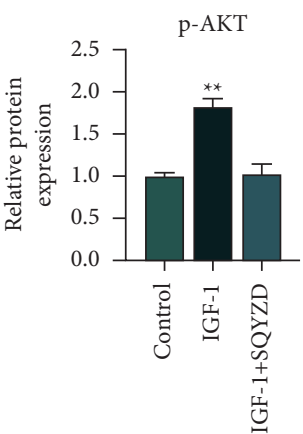

(d)

FIgURE 4: Continued. 


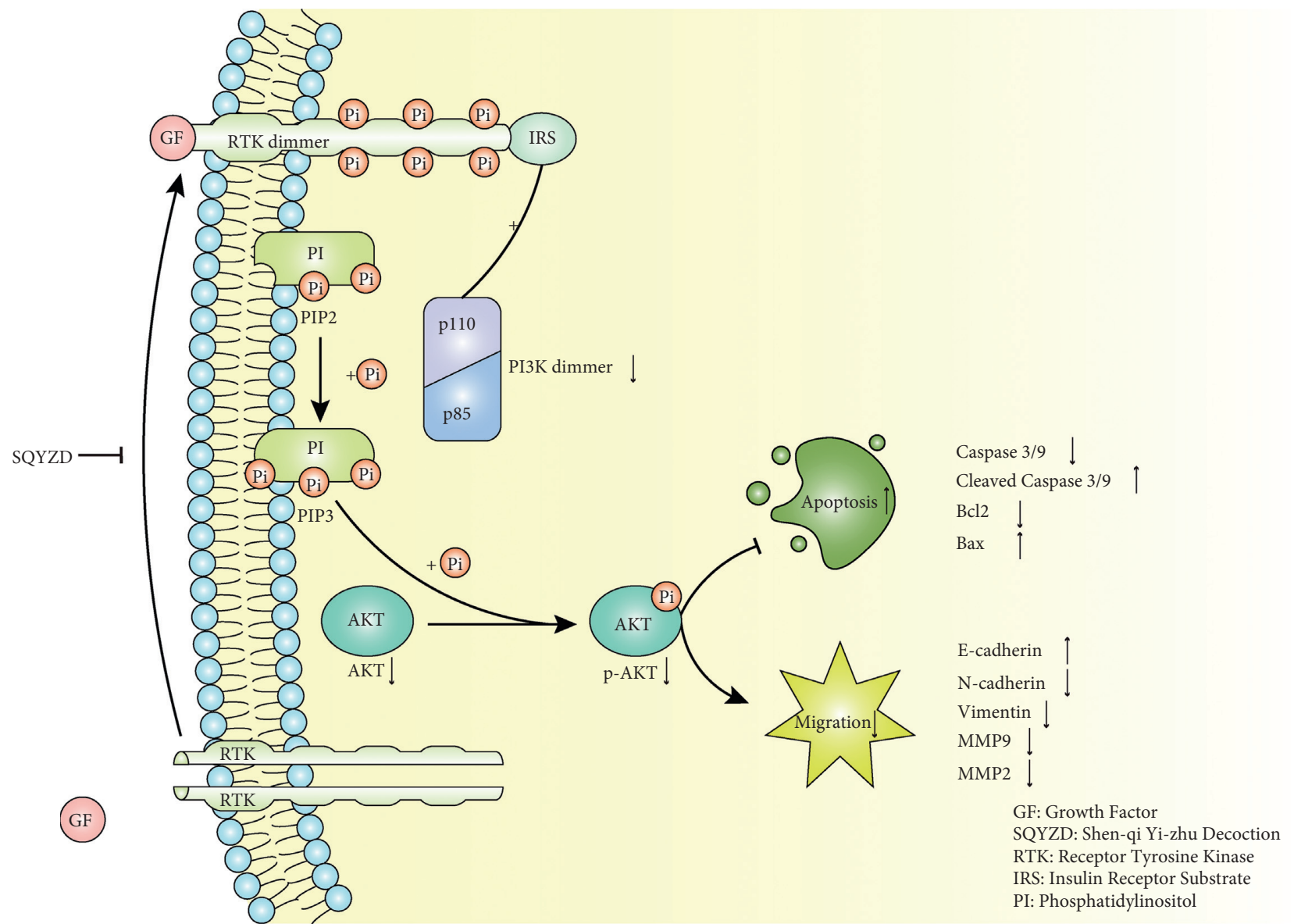

(e)

FIGURE 4: (a) Expression of PI3K/AKT/mTOR-related proteins examined using western blots after treatment with/without SQYZD (0, 2, 4, and $8 \mathrm{mg} / \mathrm{ml}$ ). (b) Expression of PI3K/AKT/mTOR-related proteins examined using western blots after treatment with $5 \mu \mathrm{M} \mathrm{IGF,} \mathrm{an} \mathrm{agonist}$ to PI3K/AKT/mTOR signaling pathway, or along with $4 \mathrm{mg} / \mathrm{ml} \mathrm{SQYZD.} \mathrm{(c)} \mathrm{Simple} \mathrm{mode} \mathrm{diagram} \mathrm{of} \mathrm{SQYZD} \mathrm{acting} \mathrm{on} \mathrm{the} \mathrm{PI3K/AKT/}$ mTOR signaling pathway.

and unclear pharmacological mechanism of action of TCMs, it is a great challenge to their pharmacological research. The development of network pharmacology provides databases and disease target prediction for TCM research, facilitates the study of active ingredients and mechanisms of TCM, and provides new ideas and methods for the development of TCM modernization.

In our study, the preliminary analysis based on network pharmacology provided a basis for subsequent studies on the pharmacodynamic ingredients and mechanisms of SQYZD. In this study, the active components of SQYZD and their molecular mechanisms in GC were predicted overall, and a variety of potential targets were explored. Meanwhile, we also identified the components in the SQYZD extract by mass spectrometry to verify the rationality of the network pharmacology results.
An approach to combine network pharmacology and experimental verification was used to predict and verify the antiGC mechanisms of SQYZD in this study. According to the results of network pharmacology, we verified the phenotype of SQYZD in promoting apoptosis and inhibiting EMT and showed its concentration-dependent features by western blot.

The PI3K/AKT/mTOR signaling pathway is an important signaling pathway in cancer, which is active in most cancers and plays an important role in cancer biology. Studies have shown that the PI3K/AKT/mTOR pathway is involved in regulating the occurrence and development of gastric cancer mediated by cell proliferation, antiapoptosis, migration, and glucose metabolism [25, 26]. According to our network pharmacology speculation, SQYZD also plays an antitumor role by inhibiting the PI3K/AKT/mTOR pathway. Through the rescue experiments, we verified that 
SQYZD can suppress antiapoptosis and EMT by inhibiting the PI3K/AKT/mTOR pathway (Figure 4(e)).

However, there are still some shortcomings in this study. The current work lacks in vivo experiments, which will be further verified in the follow-up research.

\section{Data Availability}

The data used to support the findings of this study are available from the corresponding author upon request.

\section{Disclosure}

The funders had no role in study design, data collection and analysis, the decision to publish, or the preparation of the manuscript.

\section{Conflicts of Interest}

The authors declare that they have no conflicts of interest.

\section{Authors' Contributions}

All authors made a significant contribution to the work reported, whether that is in the conception, study design, execution, acquisition of data, analysis and interpretation, or in all these areas; took part in drafting, revising, or critically reviewing the article; gave final approval of the version to be published; have agreed on the journal to which the article has been submitted; and agreed to be accountable for all aspects of the work. Shuhong Zeng and Zhibao Yu are co-first authors. Weimin Lu and Xi Zou are conjointly designated as corresponding authors.

\section{Acknowledgments}

This work was supported by the "Xu Jing-fan Academic Experience Inheritance Research Office" Construction Project of the State Administration of Traditional Chinese Medicine (Teaching and Succession Education Office of the Personnel Education Department of Traditional Chinese Medicine [2010] No. 59); the Youth Science and Technology Project of Suzhou (No. KJXW2019059); the Suzhou Science and Technology Development Plan (No. SYSD2019006); Advantageous disciplines program of Nanjing University of Chinese Medicine (Nos. ZYX03KF022 and ZYX03KF019), Science and Technology Project of Affiliated Hospital of Nanjing University of Chinese Medicine (No. Y2020CX62), State Administration of Chinese Medicine Project (No. 20085-9-3); Jiangsu Provincial Science and Technology Department Project (No. BE2019771); Jiangsu Province Postgraduate Research Innovation Program Project (No. KYCX21-1677); the Science and Technology Project of Jiangsu Provincial Administration of Traditional Chinese Medicine (No. YB201843); The Social Development Fund of Zhenjiang (No. SHW2017007), the Clinical Medicine Development Project of Jiangsu University (No. JLY20160103), and the Research Project of Cadre Health Care in Jiangsu Province (No. BJ18025).

\section{Supplementary Materials}

Supplement 1: 161 active ingredients passed the repetition, the compounds are shown in Supplement 1. Supplement 2: 63 compounds of best match score were over 70 in mzCloud are shown in Supplement 2. (Supplementary Materials)

\section{References}

[1] K. Li, A. Zhang, X. Li, H. Zhang, and L. Zhao, "Advances in clinical immunotherapy for gastric cancer," Biochimica et Biophysica Acta (BBA) - Reviews on Cancer, vol. 1876, no. 2, Article ID 188615, 2021.

[2] W. Chen, R. Zheng, P. D. Baade et al., "Cancer statistics in China, 2015," CA: A Cancer Journal for Clinicians, vol. 66, no. 2, pp. 115-132, 2016.

[3] Y. Zhang, Y. Lou, J. Wang, C. Yu, and W. Shen, "Research status and molecular mechanism of the traditional Chinese medicine and antitumor therapy combined strategy based on tumor microenvironment," Frontiers in Immunology, vol. 11, Article ID 609705, 2020.

[4] J. Xu, W. Lu, and D. Xu, "Effect of Shenqi Yizhu Decoction combined with Tegafur and Oxaliplatin on the short-term efficacy and clinical benefit of patients with advanced gastric cancer," Liaoning Journal of Traditional Chinese Medicine, vol. 45, no. 11, pp. 2325-2327, 2018, in Chinese.

[5] J. Xu and W. Lu, "Effect of Shenqi Yizhu decoction on epithelial-mesenchymal transition of gastric cancer cell through Wnt/ $\beta$-catenin signaling pathway," Jilin Journal of Chinese Medicine, vol. 38, no. 11, pp. 1311-1315, 2018, in Chinese.

[6] L. Sun, Curative Effects Evaluating of Postoperative Gastric Cancer Treated by Shenqiyizhu Decoction Combined with Chemotherapy, Nanjing University of Chinese Medicine, Nanjing, China, 2017.

[7] S. I. Berger and R. Iyengar, "Network analyses in systems pharmacology," Bioinformatics, vol. 25, no. 19, pp. 2466-2472, Oct. 2009.

[8] J. Ru, P. Li, J. Wang et al., "TCMSP: a database of systems pharmacology for drug discovery from herbal medicines," Journal of Cheminformatics, vol. 6, no. 1, p. 13, 2014.

[9] S. Y. K. Fong, A. Bauer-Brandl, and M. Brandl, "Oral bioavailability enhancement through supersaturation: an update and meta-analysis," Expert Opinion on Drug Delivery, vol. 14, no. 3, pp. 403-426, 2017.

[10] L. Guan, H. Yang, Y. Cai et al., "ADMET-score - a comprehensive scoring function for evaluation of chemical druglikeness," Medchemcomm, vol. 10, no. 1, pp. 148-157, 2019.

[11] UniProt Consortium, "UniProt: a worldwide hub of protein knowledge," Nucleic Acids Research, vol. 47, no. D1, pp. D506-D515, 2019.

[12] M. Rebhan, V. Chalifa-Caspi, J. Prilusky, and D. Lancet, "GeneCards: integrating information about genes, proteins and diseases," Trends in Genetics, vol. 13, no. 4, p. 163, 1997.

[13] J. Piñero, À Bravo, N. Queralt-Rosinach et al., "DisGeNET: a comprehensive platform integrating information on human disease-associated genes and variants," Nucleic Acids Research, vol. 45, no. D1, pp. D833-D839, 2017.

[14] A. Hamosh, A. F. Scott, J. S. Amberger, C. A. Bocchini, and V. A. McKusick, "Online Mendelian Inheritance in Man (OMIM), a knowledgebase of human genes and genetic disorders," Nucleic Acids Research, vol. 33, pp. D514-D517, 2005. 
[15] X. Chen, Z. L. Ji, and Y. Z. Chen, "TTD: therapeutic target database," Nucleic Acids Research, vol. 30, no. 1, pp. 412-415, 2002.

[16] D. S. Wishart, Y. D. Feunang, A. C. Guo et al., "DrugBank 5.0: a major update to the DrugBank database for 2018," Nucleic Acids Research, vol. 46, no. D1, pp. D1074-D1082, 2018.

[17] D. Warde-Farley, S. L. Donaldson, O. Comes et al., "The GeneMANIA prediction server: biological network integration for gene prioritization and predicting gene function," Nucleic Acids Research, vol. 38, no. 2, pp. W214-W220, 2010.

[18] D. Szklarczyk, J. H. Morris, H. Cook et al., "The STRING database in 2017: quality-controlled protein-protein association networks, made broadly accessible," Nucleic Acids Research, vol. 45, no. D1, pp. D362-D368, 2017.

[19] P. Shannon, A. Markiel, and O. Ozier, "Cytoscape: a software environment for integrated models of biomolecular interaction networks," Genome Research, vol. 13, no. 11, pp. 2498-2504, Nov. 2003.

[20] The Gene Ontology Consortium, "The gene Ontology resource: 20 years and still going strong," Nucleic Acids Research, vol. 47, no. D1, pp. D330-D338, Jan. 2019.

[21] M. Kanehisa and S. Goto, "KEGG: kyoto encyclopedia of genes and genomes," Nucleic Acids Research, vol. 28, no. 1, pp. 27-30, 2000.

[22] G. Yu, L.-G. Wang, Y. Han, and Q.-Y. He, "clusterProfiler: an $\mathrm{R}$ package for comparing biological themes among gene clusters," OMICS: A Journal of Integrative Biology, vol. 16, no. 5, pp. 284-287, 2012.

[23] Y. Chen, M. Yu, Z. Liu, Y. Zhang, Q. Li, and G. Yang, "Effects of traditional Chinese medicine combined with chemotherapy for extensive-stage small-cell lung cancer patients on improving oncologic survival: study protocol of a multicenter, randomized, single-blind, placebo-controlled trial," Trials, vol. 22, no. 1, p. 437, 2021.

[24] M. Cheng, J. Hu, and Y. Zu, "Efficacy and safety of astragaluscontaining traditional Chinese medicine combined with platinum-based chemotherapy in advanced gastric cancer: a systematic review and meta-analysis," Frontiers in Oncology, vol. 11, Article ID 632168, 2021.

[25] C. Porta, C. Paglino, and A. Mosca, "Targeting PI3K/Akt/ mTOR signaling in cancer," Frontiers in Oncology, vol. 4, p. 64, 2014.

[26] I. Pal and M. Mandal, "PI3K and Akt as molecular targets for cancer therapy: current clinical outcomes," Acta Pharmacologica Sinica, vol. 33, no. 12, pp. 1441-1458, 2012. 